\title{
ÉTUDE THÉORIQUE DE LA DIFFUSION DES ÉLEGTRONS DE HAUTS VOLTAGES
}

Par J. WINTER.

\begin{abstract}
Sommaire. - Il s'agit d'étudier la diffusion de jets électroniques de très hauts voltages, par des atomes neutres. Comme dans notre prócédent article $\left(^{1}\right)$, nous nous plaçons à un point de vue purement mathématique et avons cherché à appliquer aux électrons de très courtes longueurs d'onde, la méthode rigoureuse, appliquée généralement aux électrons lents et qui revient à suivre la propagation des ondes de de Broglie.

Pour cela nous sommes amenés à employer des expressions asymptotiques de l'argument $K r, K$ quantité de mouvement des électrons incidents, $r$ rayon de l'atome diffuseur, expressions qui représenteront, soit les éléments de décomposition de l'onde plane par la formule de Rayleigh, soit l'onde à l'intérieur de l'atome. Nous étudions les déphasages subis par les éléments de décomposition de l'onde plane, et cherchons ensuite quelles déductions on peut en tirer concernant la diffusion totale, et sa répartition angulaire.
\end{abstract}

Critique de la Méthode de Born. - Pour étudier la diffusion des électruns de hauts voltages, on emploie généralement la méthode de Born : Elle consiste à appliquer, dans les conditions que nous allons définir, le processus des approximations successives.

On cherche une solution de l'équation

$$
\Delta \Psi+K^{2} \Psi=F \Psi
$$

où $K$ représente la quantité de mouvement des électrons incidents et $F$ le potentiel perturbateur, et l'on part comme première approximation de l'onde incidente $\mathbf{e}^{-i K x}$ (unités de Hartree).

Résumons les conclusions auxquelles sont parvenus les auteurs qui ont étudié ce problème :

Pour que la méthode de Born donne une suite d'approximations $\Psi_{0}, \Psi_{1}, \Psi_{2}, \ldots$ de somme convergente, il faut: $1^{\circ}$ que le potentiel $F$ décroisse, aux grandes distances au moins comme $\frac{1}{r^{2}}$

$2^{\circ}$ Que le paramètre $K$ soit ass $\mathrm{z}$ grand. On voit même quelquefois écrire que la convergence de la méthode de Born est mesurée par le nombre $\frac{Z e E}{h v}(Z$ nombre atomique, e, $E$ charges élémentaires, $v$ vitesse des électrons incidents). En effet, on a calculé les approximations successives $\Psi_{n}$, et on a montré que $\Psi_{n}$ contenait en facteur ce nombre, élevé à la puissance $n$. (Pour ce calcul, on " interrompt 》 $F$, en supposant les charges négatives périphériques de l'atome concentrée sur une sphère).

En réalité, ces auteurs (Möller, Distel) n'ont pu donner aucune méthode utilisable pour définir la limite de validité de la première approximation de la méthode de Born. Les principales propriétés de ce développement (convergence, par rapport aux paramètres et, suivant les diverses valeurs de l'angle d'observation $\theta$ ) sont encore inconnues. Ainsi, pour des valeurs données des paramètres, on ne sait pas l'ordre de grandeur de l'erreur commise, ni la manière dont elle varie avec la direction $\theta$ où l'on observe la diffusion. Dans leur livre $\left({ }^{2}\right)$, Massey et Mott donnent bien une réponse à la première question, mais elle n'a

(') Journal de Physique, juin 1933, p. 316-323. Nous ne répétons ici ni les hypothèses, ni les résultats de cet article, dont la connaissance est nécessaire pour pouvoir comprendre celui-ci.

(2) Theory of atomic collisions. Oxford, 1933. 
aucune rigueur mathématique et semble d'application difficile. De plus, elle ne permet pas de donner la variation de l'erreur commise avec $\theta$. Notre but a donc été de former des expressions asymptotiques, permettant de retrouver la formule de Born à partir des formules rigoureuses, établies à l'aide de la décomposition de l'onde plane. Les conditions de validité de ces expressions asymptotiques sont connues, et l'on pourra trouver, en fonction de $\theta$, une limite supérieure de l'erreur commise. La marche générale consiste à : $\mathbf{1}^{\circ}$ Calculer les déphasages $\delta_{n} ; 2^{\circ}$ Simplifier l'expression du flux diffusé (ce qui donnera la formule de Born); $3^{\circ}$ Discuter l'erreur commise en fonction des paramètres et de $\theta$. Dans le présent article nous donnons le calcul des déphasages, qui permet de suivre l'allure générale du phénomène. A la fin, nous ne faisons qu'indiquer très sommairement la manière dont l'expression du flux diffusé se ramène à celle de Born : On obtient une suite de termes qui tendent vers les premiers termes d'une série convergente de somme $\frac{1}{\sin ^{2} \frac{\theta}{2}}$. Cette dernière série converge d'ailleurs d'autant plus lentement que $\theta$ est plus voisin de 0 ou $\pi$.

Nous ne faisons qu'indiquer ces derniers résultats, afin de ne pas entraîner le lecteur dans trop de discussions mathématiques. De mème, nous ne donnons que les résultats des calculs par la méthode de col, dont nous ne reprendrons pas la définition.

Les travaux de Debye $\left(^{1}\right)$. - Afin d'éviter la méthode de Born, nous allons donc suivre la méthode usuelle dans le cas où $K r<1$. En cela, nous allons suivre les idées générales indiquées en 1909 par P. Debye. Cet auteur étudiait alors la diffusion des ondes lumineuses par de petites sphères conductrices, problème analogue au nôtre, et a cherché à voir ce qui se passait lorsque la longueur d'onde de la lumière incidente était beaucoup plus petite que la sphère diffusante. Il a montré qu'il fallait employer deux sortes de formules asymptotiques, les unes valables pour des indices $n \leqslant K r$, les autres pour des indices quelconques. Nous les appellerons formules I et II. Les formules I sont les formules usuelles.

$$
\begin{aligned}
& J_{n+\frac{1}{2}}=\sqrt{\frac{2}{\pi K r}} \cos \left(K r-\frac{n+1}{2} \cdot \pi\right) \\
& H_{n+\frac{1}{2}}^{(1)}=\sqrt{\frac{2}{\pi K r}} \mathrm{e}^{i\left(K r-\frac{n+1}{2} \cdot \pi\right)} \\
& H_{n+\frac{1}{2}}^{(2)}=\sqrt{\frac{2}{\pi K r}} \mathrm{e}^{-i\left(K r-\frac{n+1}{2} \cdot \pi\right)}
\end{aligned}
$$

Enfin, les formules II s'écrivent différcmment selon que $n$ est $<,>$ ou $=$ à $K r$. Pour $n<K r$ (mais il faut malgré tout que $n$ soit grana, c'est-à-dire $\geqslant 1$ ). On a

$$
\begin{aligned}
& H_{n}^{(1)}(x)=\frac{1}{\pi} \mathbf{e}^{i x(\sin \tau-\tau \cos \tau)} \frac{\Gamma\left(\frac{1}{2}\right) \mathrm{e}^{-i \frac{\pi}{4}}}{\sqrt{\frac{x}{2} \sin \tau}} \mid \\
& H_{n}^{(2)}(x)=\frac{1}{\pi} \mathbf{e}^{-i x \cdot \sin =-\tau \cos \tau)} \frac{\Gamma\left(\frac{1}{2}\right) \mathrm{e}^{i \frac{\pi}{4}}}{\sqrt{\frac{x}{2} \sin \tau}}
\end{aligned}
$$

(1) Mathematische Annalen, t 67 (1909), p. 538; Ann. der Physik, t. 30 (1909), p. 57. 
avec

$$
\cos \tau=\frac{n}{x} \quad u<x
$$

Debye a montré que les ondes diffusées d'indices $>K r$ étaient d'intensité négligeable, et a fait une étude de la pression de radiation. Nos calculs seront plus simples que ceux de Debye, car en utilisant les résultats de notre précédent article, nons nous bornerons à calculer les déphasages des ondes extérieures.

Etude des déphasages. - Nous commencerons par faire une remarque générale :

Remarque I. - Nous voulons prolonger une onde $\Psi_{1}$ à travers une discontinuité du champ. Nous savons : $1^{\circ}$ que l'onde prolongée à la forme $c \Psi_{2} ; 2^{\circ}$ que $\Psi_{1}$ et $c \Psi_{2}^{\circ}$ transportent le même flux si $\left|\Psi_{1}\right|=\left|c \Psi_{2}\right|$

Alors il suffira que $\Psi_{1}=c \Psi_{2}$ sur la surface de discontinuité : il n'y aura pas d'onde réfléchie.

Appliquons cette remarque au cas qui nous occoupe : l'onde plane se décompose en ondes convergentes $\left(^{1}\right) H_{n+\frac{1}{2}}^{(1)}$ et divergentes $H_{n+\frac{1}{2}}^{(2)}$ telles que :

$$
H_{n+\frac{1}{2}}^{(1)}=H_{n+\frac{1}{2}}^{(2)^{*}}
$$

et de même, l'onde intérieure à la sphère perturbatrice (où nous supposons régner un potentiel de la forme $\left.\frac{Z e}{r}\right)$ en fonctions analogues $R_{n}^{(1)}$ et $R_{n}^{(2)}$, imaginaires conjuguées, transportant les mêmes flux que les $\boldsymbol{I}_{n+\frac{1}{2}}$. L'onde incidente $\boldsymbol{H}_{n+\frac{1}{2}}^{(1)}$ sera réfractée suivant $R_{n}^{(1)}$ (qui correspond au même polynôme de Legendre $\boldsymbol{P}_{n}$ ), à laquelle on superposera $R_{n}^{(2)}$, qui à son tour donnera $H_{n+\frac{1}{2}}^{(2)}$. Le déphasage $2 \delta_{n}$ subi par $H_{n+\frac{1}{2}}^{(2)}$ (voir étude précédente) sera le double de la différence de phase de $H_{n+\frac{1}{2}}^{(1)}$ et de $R_{n}^{(1)}$.

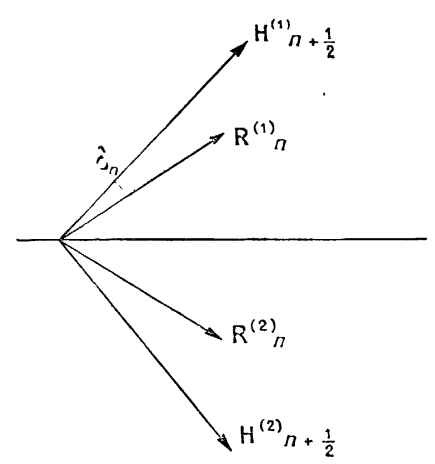

Fig. 1 .

En effet, si $\delta_{n}$ est cette différence de phase, $R_{n}^{(1)}$ sera le prolongement de $H_{n+\frac{1}{2}}^{(1)}$, si on la multiplie par $c e^{i \delta_{n}}$ ( $c$ constante réelle). La figure (1) montre qu'il en résultera pour $H_{n+\frac{1}{2}}^{(2)}$ un déphasage $2 \delta_{n}$, car la différence de phase entre $R_{n}^{(1)}$ et $R_{n}^{(\Psi)}$ est déterminée et doit rester constante.

(1) Nous n'écrivons pas le facteur, sans influence ici. 
Déphasages I. - Le paramètre $K r=\rho$ étant supposé très grand, nous partagerons les dẻphasages en 2 classes : les dèphasages I, d'indices $<\sqrt{ } \bar{\rho}$, et les déphasages II d'indices compris en $\sqrt{ } \vec{\rho}$ et $\rho$; nous montrerons que les déphasages d'indice $>\rho$ sont nuls.

La coupure $\sqrt{ } \bar{p}$ est un peu arbitraire. Les formules I exigent, pour être valables, qu'on ait $n^{2}<p$, le deuxième terme du déreloppement asymptotique différent du premier d'un facteur $\frac{n^{2}}{F}$ (si $\frac{i a Z}{\kappa}$ est très petit).

Les formules II seront encore valables pour $n=\sqrt{\rho}$ puisque $\sqrt{ } \bar{p}$ est grand, et elles coïncident pour cette valeur avec les formules I. Dans le voisinage de $n=\sqrt{ } \bar{\rho}$, les formules I et II sont équivalentes. Ne voulant pas insister sur ces considérations, nous admettrons que les formules II seront valables à partir de $V_{\bar{\rho}}$ et qu'elles se raccorderont parfaitement avec les formules I au point $n=\sqrt{ } \bar{\rho}^{-}$.

Sommerfeld et Schur $\left(^{1}\right)$ ont calculé $R_{n}^{(1)}$ et $R_{n}^{(2)}$ et trouvé

$$
\left.\begin{array}{l}
R_{n}=\frac{1}{2}\left(R_{n}^{(1)}+R_{n}^{(2)}\right) \quad R_{n}^{(1)}=R_{n}^{(2) *} \\
R_{n}^{(2)}=\frac{1}{i K r} \frac{\mathrm{e}^{-\frac{\pi}{2} \frac{Z}{a K}+\frac{i \pi n}{2}}}{\Gamma\left(n+1-i \frac{Z}{a K}\right)} \mathrm{e}^{-i\left(K r+\frac{Z}{K a} \log 2 K r\right)}
\end{array}\right\}
$$

avec $a=\frac{k^{2}}{4 \pi^{2} m e^{2}}$, rayon de la première orbite de Bohr; $Z$ nombre atomique. Avec les unités de Hartree, on aurait $a=1$.

Comparons $I$ et $I^{\prime}$. Si nous dérivons ces expressions par rapport à $r$, nous avons $\left({ }^{2}\right)$

$$
\begin{aligned}
\frac{\mathrm{d}}{\mathrm{d} r} \frac{H_{n+\frac{1}{2}}^{(1)}}{\sqrt{K r}}=K \frac{H_{n+1}^{(1)}}{\sqrt{\overline{K r}}}\left[i-\frac{1}{K r}\right] \\
\frac{\mathrm{d}}{\mathrm{d} r} R_{n}^{(1)}=K R_{n}^{(1)}\left[i-\frac{1}{K r}-\frac{Z}{a K^{2} r}\right]
\end{aligned}
$$

Mais nous devons limiter l'étendue du champ perturbateur, pour cela nous supposerons toutes les charges négatives périphériques de l'atome diffuseur concentrées sur la sphère de rayon a ; ceci est arbitraire, mais suffit pour le but que nous nous proposons : nous verrons en effet qu'en posant $r=C a$, on ne changerait rien aux résultats concernant la distribution des flux diffusés $\left({ }^{3}\right)$. Donc

$$
a=r, \frac{Z}{a K^{2} r}=\frac{Z}{\rho^{2}} . \quad \text { Si } \quad H_{n+\frac{1}{2}}^{(1)}=R_{n}^{(1)}
$$

(') Annalen der Physik, t. 4 (1930), p. 409.

(2) Les expressions asymptotiques employées sont dérivables.

(3) Ceci nous conduit à un potentiel perturbateur égal à $\frac{Z e}{r}\left(1-\frac{r}{a}\right)$, limité à $r=a$. Đans cet article, nous ne parlons pas de l'influence du facteur $1-\frac{r}{a}$; ceci revient à introduire une discontinuité de potentiel, sans signification physique, dans le but de simplifier les calculs. Les résultats essentiels obtenus ici subsistent, lorsqu'on revient à la véritable expression du potentiel, mais on obtiendra des conditions de validité plus restrictives pour la formule de Born. 
l'égalité des dérivées normales en résultera, au $2^{\text {e }}$ ordre près $\left({ }^{1}\right)$. L'emploi des formules asymptotiques limitées au premier terme nous oblige à négliger de telles quantités. Donc en vertu de la remarque I, $\delta_{n}$ sera égal à la différence de phase entre $H_{n+\frac{1}{2}}^{(1)}$ et $R_{n}^{(1)}$

$$
\delta_{n}=-\frac{Z}{a K}\left[\log 2 K r-\frac{\Gamma^{\prime}(n+1)}{\Gamma(n+1)}\right]=-\frac{Z}{\rho}\left[\log 2 p-\frac{\Gamma^{\prime}(n+1)}{\Gamma^{\prime}(n+1)}\right] .
$$

(Si nous développons J' $\left(\frac{Z}{i a K}+n+1\right)$ en série de Taylor, ce qui est licite.)

Pour $n$ assez grand $\quad \frac{\Gamma^{\prime}(n+1)}{\Gamma(n+1)}=\log n$.

Déphasages II. - Nous avons à l'aide de la méthode de Col, établi les formules II', analogues à II.

$$
\begin{aligned}
& R_{n}=\frac{1}{2}\left(R_{n}^{(1)}+R_{n}^{(2)}\right) \quad R_{n}^{(1)}=\boldsymbol{K}_{n}^{(2) *}
\end{aligned}
$$

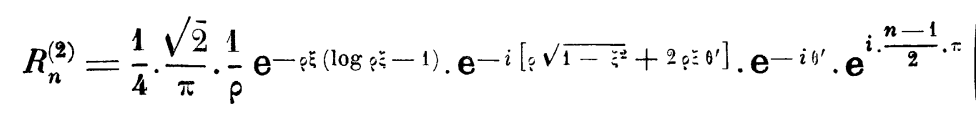

$$
\begin{aligned}
& \times \frac{1}{\sqrt{\rho \xi \sqrt{1-\xi^{2}}}} \cdot e^{-\frac{i Z}{q}\left\{\log \frac{1+\sqrt{1-\frac{\xi}{\xi}}}{\xi}-i \frac{\pi}{2}\right\}} \\
& \text { avec } \quad \xi=\frac{n}{\rho} \quad \xi \neq 0, \quad \xi \neq 1,
\end{aligned}
$$

l'angle $\theta^{\prime}$ étant défini par la relation

$$
\theta^{\prime}=\frac{1}{2} \arcsin \xi
$$

Lorsque $\xi$ tend vers 0 , on retrouve les formules $\mathrm{I}^{\prime}$, en remplaçant partout $\rho \xi$ par $n$, qu'on laissera constant.

Il faut cependant que $n$ soit grand,

Comparons II et II'; pour cela, on posera

$$
\tau=\frac{\pi}{2}-2 \theta^{\prime}
$$

le facteur imaginaire de $R_{n}^{(2)}$ deviendra (en laissant de còté l'exponentielle

$$
\begin{aligned}
& \mathrm{e}^{\left.-i \frac{Z}{i}\left\{\log \frac{1+\sqrt{1-\xi}}{\zeta}-i \frac{\pi}{2}\right\}\right)} \\
& \mathbf{e}^{-i \varphi\left[\sin \tau^{-\tau \cos \tau]}\right]} \mathbf{e}^{i \frac{\pi}{+}} \mathbf{e}^{i \frac{\tau}{2}}
\end{aligned}
$$

Dans II remplaçons $n$ par $n+\frac{1}{2}$, nous poserons $\mathrm{d} n=\frac{1}{2}$ ce qui est licite comme $n$ est grand; il faudra par suite multiplier II précisément par $\mathbf{e}^{i \frac{\tau}{2}}$.

(1) L'égalité au $2^{e}$ ordre est nécessaire puisque les déphasages sont eux-mêmes du premier ordre. Le premier terme négligé du développement asymptotique ne modifiera pas ce résultat.

(2) Whittaker, Watson, Modern analysis, p. 248. 
Donc la différence de phase entre II' et II est

$$
\delta_{n}=-\frac{Z}{\rho}\left[\log \frac{1+\sqrt{1-\xi^{2}}}{\xi}\right] \quad \text { avec } \quad \xi=\frac{n}{\rho} .
$$

Il nous reste à montrer sur les formules II et II' que l'égalité de $H_{n+\frac{1}{2}}^{(1)}$ et $R_{n}^{(1)}$ entraîne; à des termes néglizeables près, l'égalité des dérivées normales. Cette vérification se fait sans difficulté. Elle montre qu'il faut exclure un petit intervalle au voisinage de $\xi=1$.

Cet intervalle sera d'autant plus petit que p sera plus grand.

Indices voisins ou supérieurs à p. - La méthode de col nous conduit à adopter pour $\boldsymbol{R}^{(1)}$ et $\boldsymbol{R}^{(2)}$ des expressions prises au voisinage d'une même valeur de la variable complexe d'intégration et en ce point, un calcuI simple montre que les déphasages sont nuls. Les $\delta_{n}$ s'annulent tous, ce que laissait prévoir le résultat obtenu par Debye.

Conclusion. - Si $\rho$ est grand et si $\frac{Z}{\rho}$ est petit l'expression du déphasage en fonction de l’indice $n$ sera donnée asymptotiquement par

$$
\begin{array}{ll}
n<\sqrt{\rho} & \delta_{n}=-\frac{Z}{\rho}\left[\log 2 \rho-\frac{\Gamma^{\prime}(n+1)}{\Gamma(n+1)}\right] \\
\sqrt{\rho}<n<\rho & \delta_{n}=-\frac{Z}{\rho}\left[\log \frac{1+\sqrt{1-\xi^{2}}}{\xi}\right] \quad \text { avec } \quad \xi=\frac{n}{\rho} \\
\rho \leqslant n & \delta_{n}=0 .
\end{array}
$$

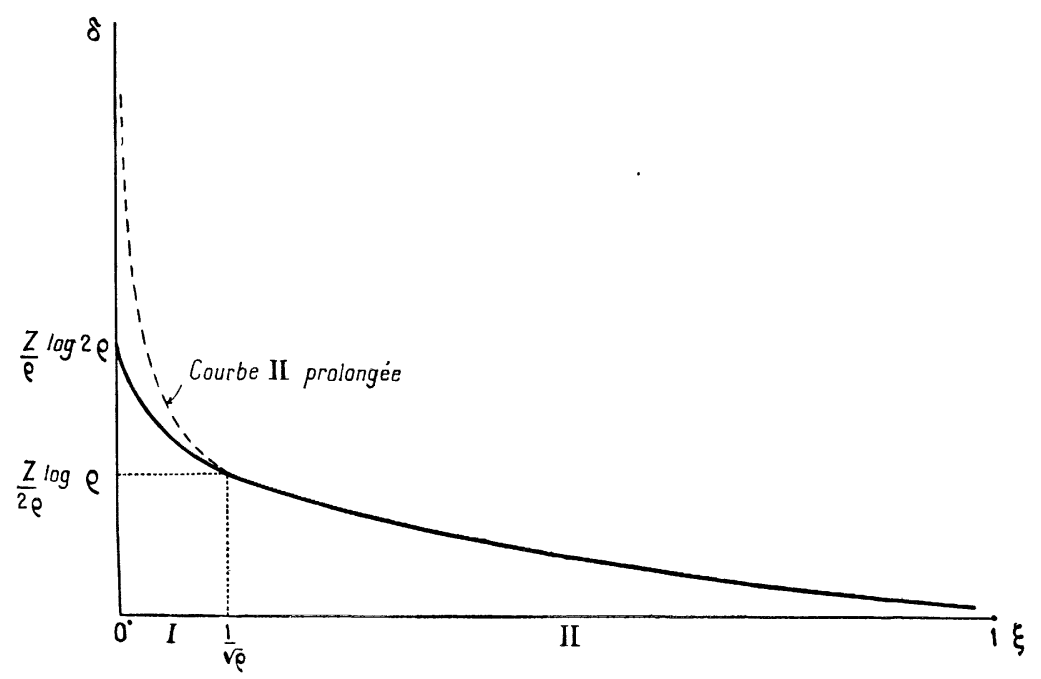

Fig. 2.

La dérivée de $\log \frac{1+\sqrt{1-\xi^{2}}}{\xi}$ est $-\frac{1}{\xi \sqrt{1-\xi^{2}}}$. La fonction II décroît de $+\infty$ à 0 (si nous changeons les signes de tous les déphasages), quand $\zeta$ varie de 0 à 1.

$$
\text { Pour } \quad n=\sqrt{\rho}, \quad \xi=\frac{1}{\sqrt{\rho}}, \quad \delta=\frac{Z}{\rho}[\log 2 \rho-\log \sqrt{\rho}] \sim \frac{Z}{2 \rho} \log \rho \text {. }
$$


La fonction I décroît depuis la valeur $\frac{Z}{\rho} \log 2 p$ jusqu'à la valeur $\frac{Z}{2 p} \log \rho($ pour $n=\sqrt{p})$. La dérivée à l'origine est infinie.

On a donc l'allure indiquée figure 2.

Lorsque $\rho$ croît. $1^{\circ}$ La fonction II représentera les $\rho$ dans un intervalle qui se rapprochera de plus en plus des extrémités $\xi=0$ et $\zeta=1$.

$2^{\circ}$ Le déphasage maximum, qui est voisin de $\frac{z}{\rho} \log \rho$ tend vers 0 .

Flux total diffusé. - Il est donné par l'expression ( $\left.{ }^{1}\right)$

$$
\Phi=\sum_{n=0}^{i} \frac{4 \pi}{K^{2}}(2 n+1) \delta_{n}^{2}
$$

si les $\delta$ sont assez petits pour être remplacés par les sinus ou bien

$$
\Phi=\frac{4 \pi}{K} \times 2 \Sigma \xi \delta_{\xi}^{2}
$$

en écrivant $\delta_{\xi}$ pour $\delta_{n}$ et

$$
\Phi=8 \pi \int_{0}^{1} ? \delta \Sigma^{2} \mathrm{~d} \xi
$$

et en posant

$$
\begin{gathered}
\delta_{\xi}=\frac{Z}{\rho} \varphi(\xi), \\
\Phi=\frac{8 \pi Z^{2}}{K^{2}} \int_{0}^{1} \xi \vartheta^{2}(\xi) \mathrm{d} \xi
\end{gathered}
$$

avec

$$
\begin{array}{ll}
\varphi(\xi)=\left[\log 2 \rho-\frac{\Gamma^{\prime}(\rho \xi+1)}{\Gamma(\rho \bar{\xi}+1)}\right]=[\log 2 \rho-\log n] & \xi<\sqrt{\rho} \\
\varphi(\xi)=\log \frac{1+\sqrt{1-\xi 2}}{\xi} & \sqrt{ } \bar{\rho}<\zeta<1 .
\end{array}
$$

L'intervalle correspondant à I tendra vers 0 si $\rho \rightarrow \infty$ et on pourra écrire asymptotiquement

$$
\Phi=\frac{8 \pi Z^{2} \varepsilon}{K^{2}} \quad \text { avec } \quad \varepsilon=\int_{0}^{1} \xi\left[\log \frac{1+\sqrt{1-\xi^{2}}}{\zeta}\right]^{2} \mathrm{~d} \xi .
$$

$\varepsilon$ est donc un facteur numérique déterminé.

Distribution angulaire du flux diffusé. - Nous avons montré que le flux diffusé, compris entre les cônes d'ouverture $\theta_{1}$ et $\theta_{2}(\theta=0$ est la direction de propagation des électrons) est donné par

$$
\begin{aligned}
\Phi_{\theta_{1}}^{\theta_{2}}= & -\frac{\pi}{K^{2}} \int_{\theta_{2}}^{\theta_{1}}\left\{\sum_{n=0}^{\rho} \sum_{n^{\prime}=0}^{\rho}(2 n+1)\left(2 n^{\prime}+1\right) P_{n}(\cos \theta) P_{n^{\prime}}(\cos \theta) \sin ^{2}\left(\partial_{n}-\delta_{n^{\prime}}\right)\right\} \sin \theta \mathrm{d} \theta \\
& +\frac{\pi}{K^{2}} \int_{\theta_{2}}^{\theta_{1}}\left\{\sum_{P=0}^{\dot{\rho}}(2 P+1) \sin ^{2} \delta_{P} P_{P}(\cos \theta)\left(\boldsymbol{P}_{\rho}^{\prime}+1(\cos \theta)+P_{\rho}^{\prime}(\cos \theta)\right\} \sin \theta \mathrm{d} \theta .\right.
\end{aligned}
$$

(1) Unité de Hartree, nous posons à partir d’ici $r=a=1$. 
assimilons les sinus aux arcs et mettons en facteur $\frac{z^{2}}{\rho^{2}}$ II vient après simplifications.

$$
\Phi_{\theta_{2}}^{\theta_{1}}=\frac{2 \pi Z^{2}}{K^{4}} I_{\theta_{1}}^{\theta_{2}} \quad \text { avec } \quad I_{\theta_{1}}^{\theta_{2}}=\int_{\theta_{1}}^{\theta_{2}}\left[\sum_{n=0}^{-}(2 n+1) p_{n} P_{n}(\cos \theta)\right]^{2} \sin \theta \mathrm{d} \theta .
$$

$\varphi$ ayant la même signification qu'au dernier paragraphe.

On peut évaluer $\Sigma(2 n+1) \varphi_{n} P_{n}$ à l'aide de la transformation d'Abel (1); il viendra

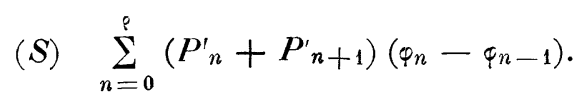

Comme $P_{n}^{\prime}(\cos \theta) \sim \frac{\sqrt{n}}{\sqrt{\sin \theta}} \cos (n \theta+\beta)$ pour de grandes $n, \beta$ étant un angle constant. Adoptons pour les $\varphi_{n}$ les expressions (I) et sommons de $n=0$ à $n=\infty$; comme $\varphi_{n}-\varphi_{n+1}=\frac{1}{n+1}$ on a une série convergente partout, sauf aux voisinages de $\theta==0$ et $\theta=\pi$. Nous avons démontré que la somme de cette série, est égale à $\frac{1}{\sin ^{2}\left(\frac{\theta}{2}\right)}$. (La démonstration fait intervenir à nouveau la transformation d'Abel, et de plus la formule de Heine, pour le développement en polynômes de Legendre des fonctions de la forme $\frac{1}{A-x}$. Nous passerons cette démonstration ici.) Ainsi la formule en $\frac{1}{\sin ^{4}\left(\frac{\theta}{2}\right)}$ exige : $1^{\circ}$ que $\rho$ soit $\geqslant 1 ; 2^{\circ} \frac{Z}{\rho} \leqslant 1$. Cette deuxième hypothèse est nécessaire pour qu'on puisse remplacer les $\sin \left(\delta_{n}-\delta_{n^{\prime}}\right) \operatorname{par}\left(\delta_{n}-\delta_{n^{\prime}}\right)$ et pour qu'on puisse développer $\Gamma\left(n+1+\frac{Z}{i p}\right)$. La formule de Born est bien valable asymptotiquement et il n'est pas nécessaire de calculer l'approximation suivante pour connaitre sa limite de validité : Il suffit de savoir jusqu'à quel terme $n$ on peut appliquer les formules I et quel sera le reste correspondant de la série $\left(S^{\prime}\right)$ pour les diverses valeurs de l'angle $\theta\left({ }^{2}\right)$. On voit de plus qu'en posant $r=c a$, rien ne serait changé à l'expression du flux diffusé latéralement.

La presque totalité du flux diffusé est comprise dans un petit cône entourant l'axe $\theta=0$, ce qui correspond au fait intuitif qu'un pinceau d'électrons de plus en plus rapides est de moins en moins déformé.

Remarque. - Il ne faut pas confondre ce problème $(r=a$, champ perturbateur limité) avec celui traité par Gordon, Sommerfeld et où le champ est illimité. Alors $r$ est

(1) Voir Lebesgue, Leçons sur les séries trigonométriques, Gauthier-Villars 1906, p. 38 et suiv.

(2) Il faut étudier le reste de la série $\frac{1}{\sin ^{2}\left(\frac{\theta}{2}\right)}$ et le reste de $S$. Lorsqu'on porte dans $S$ les valeurs (II) pour les $\varphi_{n}$, il apparait une difficulté au voisinage de $n=\rho$. On ne peut pas donner de limite supérieure du reste. Cette difficulté tient à la discontinuité de potentiel artificiellement introduite, et disparait lorsque l'on tient compte du facteur $\left(1-\frac{r}{a}\right)$. 
' $K$ étant quelconque. Les déphasages peuvent devenir infinis et le problème est complètement différent.

En terminant, nous remercions infiniment M. L. de Broglie qui, par son aide constante, nous a permis de pousser ce travail jusqu'au bout.

Manuscrit reçu le 3 octobre 1933. 